\title{
ON THE MEAN CURVATURE OF SUBMANIFOLDS OF EUCLIDEAN SPACE
}

\author{
BY BANG-YEN CHEN ${ }^{1}$
}

Communicated by S. Sternberg, April 12, 1971

Let $x: M^{n} \rightarrow E^{m}$ be an immersion of an $n$-dimensional manifold $M^{n}$ in a euclidean space $E^{m}$ of dimension $m(m>n>1)$, and let $\nabla$ and $\nabla^{\prime}$ be the covariant differentiations of $M^{n}$ and $E^{m}$, respectively. Let $u$ and $v$ be two tangent vector fields on $M^{n}$. Then the second fundamental form $h$ is given by

$$
\nabla_{u}^{\prime} v=\nabla_{u} v+h(u, v) .
$$

If $\left\{\boldsymbol{e}_{1}, \cdots, \boldsymbol{e}_{n}\right\}$ is an orthonormal basis in the tangent space $T_{p}(M)$ at $p \in M^{n}$, then the mean curvature vector $H(p)$ at $p$ is given by

$$
H(p)=(1 / n) \sum_{i=1}^{n} h\left(e_{i}, e_{i}\right) .
$$

Let $\langle$,$\rangle denote the scalar product of E^{m}$. If there exists a function $f$ on $M$ such that $\langle h(u, v), H\rangle=f\langle u, v\rangle$ for all tangent vector fields $u, v$ on $M^{n}$, then $M^{n}$ is called a pseudo-umbilical submanifold of $E^{m}$. If the covariant derivative of $H$ in $E^{m}$ is tangent to $x\left(M^{n}\right)$ everywhere, then $H$ is said to be parallel in the normal bundle. In [2], [3], the author proved that if $M^{n}$ is closed, then the mean curvature vector $H$ satisfies

$$
\int_{M^{n}}\langle H, H\rangle^{n / 2} d V \geqq c_{n},
$$

where $d V$ denotes the volume element of $M^{n}$ and $c_{n}$ is the area of the unit $n$-sphere. The equality sign of (3) holds when and only when $M^{n}$ is imbedded as a hypersphere in an $(n+1)$-dimensional linear subspace of $E^{m}$. It is interesting to know whether the inequality (3) can be improved for some special submanifolds of $E^{m}$.

The main purpose of this paper is to announce some results in this direction together with some results on pseudo-umbilical submanifolds. Details will appear elsewhere.

\footnotetext{
AMS 1970 subject classifications. Primary 53A05, 53A10, 53B25; Secondary $53 \mathrm{C} 40$.

Key words and phrases. Mean curvature vector, minimal surface, pseudo-umbilical submanifold, Clifford torus, $\alpha$ th curvatures of first and second kinds.

1 This work has been supported in part by NSF Grant GU-2648.
} 
By studying the behaviors of the mean curvature vector $H$ we can prove

LEMma 1. The position vector field $\boldsymbol{X}$ of $M^{n}$ in $E^{m}$ is parallel to the mean curvature vector $H$ when and only when $M^{n}$ is either a minimal submanifold of $E^{m}$ or a minimal submanifold of a hypersphere of $E^{m}$ centered at the origin.

By using Lemma 1, we can prove

Proposition 1 (YANo-Chen [5]). $M^{n}$ is a pseudo-umbilical submanifold of $E^{m}$ such that the mean curvature vector $H$ is parallel in the normal bundle when and only when $M^{n}$ is either a minimal submanifold of $E^{m}$ or a minimal submanifold of a hypersphere of $E^{m}$.

If the codimension is equal to 2 , then a pseudo-umbilical submanifold of $E^{m}$ with constant mean curvature is always a pseudo-umbilical submanifold such that the mean curvature vector is parallel in the normal bundle. Hence we have

Proposition 2. $M^{n}$ is a pseudo-umbilical submanifold of $E^{n+2}$ with constant mean curvature when and only when $M^{n}$ is either a minimal submanifold of $E^{n+2}$ or a minimal hypersurface of a hypersphere of $E^{n+2}$.

Let $F$ be a field and $H_{i}\left(M^{n}, F\right)$ denote the $i$ th cohomology group of $M^{n}$ over the field $F$. Let $\beta\left(M^{n}\right)=\max \left\{\sum_{i=0}^{n} \operatorname{dim} H_{i}\left(M^{n}, F\right) ; F\right.$ fields $\}$. Then, by verifying the properties of the length of second fundamental form $h$, we can prove

TheOREM I. Let $M^{n}$ be an $n$-dimensional closed manifold immersed in $E^{m}$ with nonnegative scalar curvature. Then we have

$$
\int_{M^{n}}\langle H, H\rangle^{n / 2} d V>a \beta\left(M^{n}\right),
$$

where

$$
\begin{aligned}
a & =\left(4 n^{n}\right)^{-1 / 2} c_{n}, \quad \text { if } n \text { is even, } \\
& =\left(2 n^{n} c_{m-n-1} c_{m+n-1}\right)^{-1 / 2}\left(c_{2 n}\right)^{1 / 2} c_{m-1}, \quad \text { if } n \text { is odd. }
\end{aligned}
$$

TheOREM II. Let $M^{2}$ be a flat torus in $E^{4}$. Then we have

$$
\int_{M^{2}}\langle H, H\rangle d V \geqq 2 \pi^{2} .
$$

Then the equality sign of (6) holds when and only when $M^{2}$ is a Clifford torus in $E^{4}$.

Proof (SKeTCH). The proof of (6) follows from a direct computation of the first curvature of second kind, $\lambda_{1}(p)$, (for the definition, 
see [1]) and the relations between the mean curvature and $\lambda_{1}$. If the equality of (6) holds, then we can prove that $M^{2}$ is a minimal surface of a 3 -sphere in $E^{4}$. From this we see that $M^{2}$ is a Clifford torus in $E^{4}$. The converse of this is trivial.

For each unit normal vector e to $x\left(M^{n}\right)$ at $x(p)$, let $h_{\mathbf{e}}$ be the linear transformation from the tangent space $T_{p}(M)$ into itself defined by

$$
\left\langle h_{e}(u), v\right\rangle=\langle h(u, v), e\rangle
$$

for all tangent vectors $u, v$ at $p$. Let $K(p, \boldsymbol{e})=\operatorname{det}\left(h_{\boldsymbol{e}}\right)$. Then $K(p, \boldsymbol{e})$ is called the Lipschitz-Killing curvature at $(p, e)$. By deriving some integral formulas for the $\alpha$ th curvatures of first and second kinds (for the definitions, see [1]), we can prove

THEOREM III. Let $M^{2}$ be an oriented closed surface in $E^{m}$. If $M^{2}$ is contained in a hypersphere of $E^{m}$, then $M^{2}$ is a pseudo-umbilical surface of $E^{m}$ when and only when the Lipschitz-Killing curvature in the unit direction of the mean curvature vector $H$ is maximal over the fibre of the unit normal bundle $B_{v}, B_{v}=\left\{(p, e): p \in M^{2}\right.$, e a unit normal vector in $E^{m}$ at $\left.x(p)\right\}$.

THEOREM IV (ADDED IN PROOF). The Veronese surface in $E^{5}$, the generalized Clifford tori in $E^{n+2}$ and the $n$-sphere in $E^{n+p}$ are the only closed pseudo-umbilical submanifolds $M^{n}$ of $E^{n+p}$ with mean curvature vector nowhere zero satisfying

$$
R \geqq \frac{n(p-1)\langle H, H\rangle}{2 p-3}\left[(n-1)\left(\frac{2 p-3}{p-1}\right)-1\right]
$$

where $R$ denotes the scalar curvature of $M^{n}$.

The proof of this theorem will appear in a forthcoming paper "Pseudo-umbilical submanifolds in a Riemannian manifold of constant curvature. II".

\section{REFERENCES}

1. B.-y. Chen, On an inequality of T. J. Willmore, Proc. Amer. Math. Soc. 26 (1970), 473-479.

2. - On an inequality of mean curvatures of higher degree, Bull. Amer. Math. Soc. 77 (1971), 157-159.

3. - On the total curvature of immersed manifolds. I. An inequality of FenchelBorsuk-Willmore, Amer. J. Math. 93 (1971), 148-162.

4. - On the total curvature of immersed manifolds. II. Mean curvature and length of second fundamental form (to appear).

5. K. Yano and B.-y. Chen, Minimal submanifolds of a higher dimensional sphere, Tensor (to appear).

Michigan State University, East Lansing, Michigan 48823 